\title{
POLIFARMÁCIA ENTRE IDOSOS DE UMA UNIDADE DE SAÚDE DA FAMÍLIA: UM RELATO DE EXPERIÊNCIA MULTIPROFISSIONAL
}

\author{
POLYPHARMACY AMONG ELDERLY PEOPLE FROM A FAMILY HEALTH UNIT: \\ A MULTIPROFESSIONAL EXPERIENCE REPORT
}

\author{
Amanda Maria Villas Bôas Ribeiro, Bruna Santos de Oliveira, Patrícia Sodré Araújo, Jesica \\ Tatiana Ponce, Helena Patáro de Oliveira Novaes
}

Universidade Federal da Bahia,

\begin{abstract}
This study aims to describe the performance of a multiprofessional health team with elderly people in polypharmacy and with difficulties in pharmacotherapeutic adherence, belonging to the area covered by a Family Health Unit (FHU) in a municipality in Bahia. This is an experience report, with a descriptive approach, of the performance of a multiprofessional health team composed of the Family Health team, the Family Health Center and Family Health residents with elderly people in polypharmacy and difficulty adherence to drug treatment in the area enrolled in a Family Health Unit in a municipality in Bahia. The elderly in general had difficulty understanding the guidelines for following the therapeutic project, as well as failures in the management of their health condition, medication adherence and self-care deficit, such as the administration of drugs and their storage. Such factors are mainly related to social and economic conditions, low education and cognitive problems. The realization of this experience allowed us to reflect on the importance of daily clinical practice in Primary Health Care in preventing damage caused by excess medication, called quaternary prevention, in the reality of the elderly in polypharmacy.
\end{abstract}

Keywords: Aged. Pharmaceutical Services. Patient Care Team.
Resumo

O presente estudo tem como objetivo descrever a atuação de uma equipe multiprofissional em saúde junto a idosos em polifarmácia e com dificuldades de adesão farmacoterapêutica, pertencentes à área de abrangência de uma Unidade de Saúde da Família de um município baiano. Trata-se de um relato de experiência, de abordagem descritiva, da atuação de uma equipe multiprofissional de saúde composta pela equipe de Saúde da Família, o Núcleo Saúde da Família e residentes em Saúde da Família junto a idosos em polifarmácia e dificuldade de adesão ao tratamento medicamentoso da área adscrita de uma Unidade de Saúde da Família de um município baiano. Os idosos no geral apresentaram dificuldade de compreensão das orientações para seguimento do projeto terapêutico, bem como falhas no manejo de sua condição de saúde, adesão medicamentosa $e$ déficit de autocuidado, tais como a administração de medicamentos e seu armazenamento. Tais fatores estão relacionados, principalmente, às condições sociais, econômicas, baixa escolaridade e problemas cognitivos. A realização desta experiência permitiu refletir sobre a importância da prática clínica diária na Atenção Primária em saúde na prevenção de danos causados pelo excesso de medicamentos, denominada prevenção quaternária, na realidade do idoso em polifarmácia.

Palavras-chave: Idosos. Cuidados farmacêuticos. Equipe Multiprofissional. 
O envelhecimento é um processo natural do ciclo de vida que se caracteriza por mudanças fisiológicas, físicas, psicológicas e socioeconômicas que impactam direta e indiretamente na qualidade da saúde de cada indivíduo, por estar correlacionado aos modos de viver e andar da vida dos sujeitos e seu processo saúde, adoecimento e cuidado. ${ }^{1,2}$

O envelhecimento populacional brasileiro e mundial é uma tendência crescente que tem ocorrido ao longo de décadas, em virtude do aumento da expectativa de vida resultado dos avanços tecno-científicos e melhoria das condições de vida de modo geral. ${ }^{3,4}$

Há estimativas de que o Brasil conta com cerca de 210,1 milhões de habitantes, sendo que destes aproximadamente 29 milhões são idosos. $^{5}$ Tais dados propõem uma série de desafios atuais às políticas públicas de saúde visto que a rede de atenção à saúde não está suficientemente preparada para responder às necessidades e demandas dessa população específica, mais susceptível ao processo de adoecimento crônico. ${ }^{6}$

Nesse contexto de mudanças demográficas e epidemiológicas há um aumento da prevalência de Doenças Crônicas Não Transmissíveis (DCNT) nessa população, uma vez que os idosos possuem particularidades fisiológicas que culminam com a sua elevada ocorrência. 0 acometimento dos idosos por DCNT pode provocar limitações na funcionalidade global do idoso que se relaciona a capacidade de gerir sua própria vida e cuidado, ou seja, sua autonomia e independência. $6,7,8$

Nesse sentido, a população idosa com doenças crônicas é envolvida comumente por tratamentos farmacológicos de uso prolongado ocasionando maior uso de medicamentos e consequentemente maior ocorrência de polifarmácia., ${ }^{9,10}$ Vale ressaltar que a farmacoterapia age como um fator que visa garantir um atendimento mais integral ao indivíduo e auxiliar na otimização do tratamento, porém o uso inadequado dos medicamentos pode causar sérios danos à saúde, além de favorecer o surgimento de reações adversas, redução da efetividade terapêutica, iatrogenia no usuário, acarretando a inserção de mais medicamentos na sua terapia. ${ }^{7,8,9}$

A Polifarmácia é denominada como a administração de 5 ou mais medicamentos de maneira concomitante que muitas das vezes é ocasionada por comorbidades e a automedicação. Essa prática é observada nas diversas faixas etárias da população, em especial em idosos em virtude dos medicamentos prescritos advindos do surgimento de várias doenças, realização de consultas com diversos profissionais prescritores, automedicação e até mesmo substituição dos medicamentos por tratamentos alternativos como de fitoterápicos e plantas medicinais, sem orientação adequada de um profissional de saúde. ${ }^{4}$

Entende-se que a atenção à saúde tem impulsionado a construção cotidiana da cultura da medicalização social, com seus excessos de prescrições medicamentosas e de exames diagnósticos, em torno da lógica de consumo, dependência, alienação e massificação. Nessa perspectiva, a atuação dos profissionais de saúde deve ter um enfoque na prevenção quaternária, ou seja, buscando identificar pessoas em risco de medicalização excessiva e desenvolver ações de proteção contra novas intervenções desnecessárias, evitando danos iatrogênicos. ${ }^{11}$

A população idosa, nesse sentido, é um grupo mais suscetível à medicalização excessiva, problemas relacionados à terapêutica e iatrogenia, uma vez que há o uso concomitante de diversos medicamentos prescritos, automedicação, e uso de terapias alternativas como plantas medicinais propiciando mais ainda o surgimento das reações adversas a medicamentos, interações medicamentosas e intoxicação por medicamentos. ${ }^{12}$

Sabe-se de uma significativa parcela de idosos reside sozinho e/ou possui um arranjo familiar disfuncional, atrelado a isso, a crescente perda da funcionalidade no indivíduo idoso favorece a dependência total ou parcial de terceiros para realização de suas atividades diárias. Tais aspectos em graus diferenciados exercem influência no transcorrer da terapêutica, criando obstáculos e dificuldade na adesão à terapia medicamentosa. ${ }^{13}$

Nesse contexto, o profissional de saúde tem um papel fundamental na redução de problemas relacionados ao uso de medicamentos, com destaque para o farmacêutico, profissional habilitado e com competências técnicas para atuar na adesão ao tratamento medicamentoso e no uso racional de medicamentos. ${ }^{14}$

A Atenção Básica ( $A B)$ é conhecida como uma das portas de acesso ao Sistema Único de Saúde (SUS), assim, os profissionais atuantes nesse nível de atenção são atores importantes no processo de produção do cuidado ao indivíduo haja vista que atua de forma mais próxima ao usuário, conhecendo suas necessidades de saúde e contexto socioeconômico e cultural. ${ }^{15}$ 
Por sua vez, a atuação multiprofissional favorece um cuidado integral ao indivíduo, sua família e comunidade, observando todo o contexto social, econômico e ambiental em que ele está inserido, fortalecendo o vínculo, promovendo acolhimento aos seus problemas e necessidades de saúde, bem como e coordenando o cuidado dentro da Rede de Atenção em Saúde (RAS). ${ }^{14}$

Assim, faz-se necessário a intervenção multiprofissional à idosos em polifarmácia, a fim de promover com ações para a otimização de sua farmacoterapia, o uso seguro e racional de medicamentos.

A partir do exposto, o presente estudo tem como objetivo descrever a atuação de uma equipe multiprofissional em saúde junto a idosos em polifarmácia e com dificuldades de adesão farmacoterapêutica, pertencentes à área de abrangência de uma Unidade de Saúde da Família (USF) de um município baiano.

\section{Metodologia}

Trata-se de um estudo qualitativo e descritivo do tipo relato de experiência (RE). Para fins deste estudo compreende-se que o RE interliga processos e saberes através de uma narrativa científica desempenhando uma experiência singularizada de uma determinada prática em seu território em dado tempo histórico ${ }^{16}$. Este relato buscou descrever a atuação de uma equipe multiprofissional de saúde composta pela equipe de Saúde da Família, o Núcleo Saúde da Família (NASF) e residentes em Saúde da Família junto a idosos em polifarmácia e dificuldade de adesão ao tratamento medicamentoso da área adscrita de uma Unidade de Saúde da Família (USF) de um município baiano.

Inicialmente foram realizadas reuniões entre as equipes de Saúde da Família e o NASF para solicitar aos Agentes Comunitários de Saúde (ACS) que identificassem em suas microáreas de abrangência os usuários da unidade que contemplasse os seguintes critérios: Idosos em polifarmácia e comorbidades associadas, com dificuldade de adesão à terapêutica medicamentosa, que residem sozinhos e/ou necessidades especiais ou transtorno mental.

Em seguida, realizou-se análise da história de vida pregressa e condições de saúde atuais de cada caso individualmente, a partir da leitura dos prontuários dos pacientes e discussão multiprofissional.
Após essa etapa, foram agendadas visitas domiciliares (VD) para os idosos respeitando um fluxo (figura 1) criado pelos profissionais residentes com vistas ao alcance da adesão terapêutica desses idosos em polifarmácia.

As visitas domiciliares (VD) foram planejadas para ocorrer em quatro encontros. O primeiro foi realizado pela profissional residente em Farmácia e o ACS da área, com avaliação farmacêutica, investigando o estado clínico atual, percepção geral e qualidade de vida e contato inicial com o usuário para a construção e fortalecimento do vínculo do usuário com a equipe do projeto. Nesse momento, foram realizadas intervenções pontuais a respeito de problemas na qualidade dos medicamentos utilizados, como a utilização de medicamentos fora do prazo da validade e armazenamento incorreto. A segunda VD contou com a participação da profissional residente enfermeira, ACS, assistente social e terapeuta ocupacional para a análise do contexto sócio econômico, familiar, avaliação cognitiva com auxílio do Mini Exame do Estado Mental dos idosos selecionados.

Com a realização do segundo encontro, os usuários foram observados de uma forma mais integral, analisando seu contexto econômico, acesso e encaminhamento a outros dispositivos da Rede para avaliação da condição cognitiva e visual.

Após isso, a equipe multiprofissional planejou intervenções para a melhor adesão a farmacoterapia, colocando o usuário como protagonista do seu cuidado, fortalecendo a individualidade do sujeito e humanizando a relação profissional - paciente. As intervenções englobam a realização de exames laboratoriais, encaminhamento para consultas médicas, inserção dos usuários nos grupos e oficinas da unidade, alterações na farmacoterapia através do diálogo com o médico e o melhor acesso aos medicamentos, utilizando a rede de saúde do município e estado para aquisição dos fármacos.

A terceira VD teve o objetivo de executar o plano de cuidado com o propósito de atender às necessidades relacionadas à farmacoterapia que os pacientes apresentaram. A partir da elaboração do um plano de cuidado e manejo foi possível ao farmacêutico propor soluções ou mesmo prevenir os problemas relacionados à farmacoterapia identificados, ressalta-se que as intervenções propostas para solucionar e/ou prevenir tais problemas foram pactuadas com o paciente incluindo este no processo de tomada de decisão. O farmacêutico procedeu para cada intervenção de acordo com as necessidades 
relacionadas à farmacoterapia. Detalhar nesta etapa as intervenções que realizou, analisando suas dificuldades e mudando conforme necessário.

Na quarta VD foi realizado o monitoramento das ações no último encontro onde foram discutidas juntos com o usuário e a equipe a avaliação dos ciclos e os obstáculos encontrados para a otimização da farmacoterapia.

Com isso, o quarto encontro foi marcado com a avaliação e monitoramento do plano terapêutico produzido. Foi possível observar mudanças positivas em relação ao paciente, como por exemplo, maior compreensão da sua condição de saúde e do seu projeto terapêutico, maior acessibilidade aos medicamentos, potencialização de sua autonomia, fortalecimento do vínculo do usuário com a sua equipe de referência, melhorias na continuidade do cuidado com adesão do paciente à terapêutica proposta, reconhecendo-o como protagonista do cuidado.

Figura 1 - Fluxograma para atuação multiprofissional na intervenção com idosos em polifarmácia.

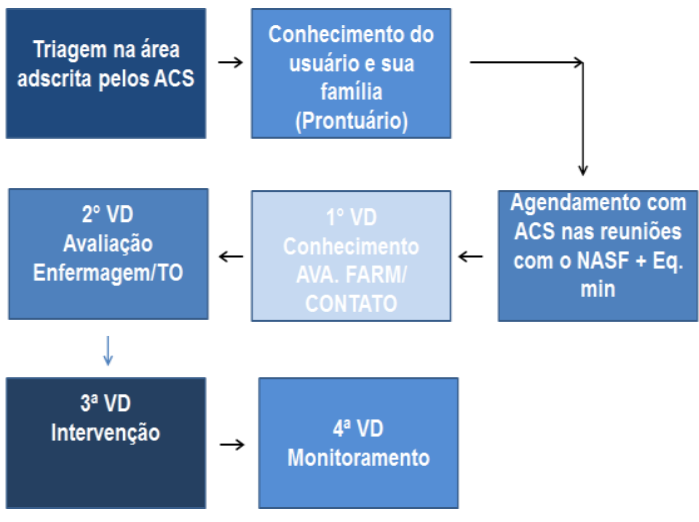

Fonte: Autoria própria.

\section{Resultados e Discussão}

Foram realizadas um total de 86 visitas domiciliares, em que foi possível perceber a vulnerabilidade dos idosos aos Problemas Relacionados a Medicamentos (PRM), relacionados à efetividade, necessidade e segurança.

Os usuários atendidos apresentaram condições de saúde como doenças cardiovasculares, doenças respiratórias crônicas, diabetes mellitus e neoplasias. Essas condições estavam relacionadas, principalmente, ao histórico familiar, a inatividade física e sedentarismo, a ingestão de bebidas alcoólicas e tabagismo. Os achados dessa experiência dialogam com alguns estudos que demonstram a relação de polifarmácia em idosos com o diagnóstico de DCNT e a inserção de medicamentos de uso crônico. ${ }^{1,17}$

$\mathrm{Na}$ realização dos atendimentos, percebeu-se que alguns pacientes apresentaram déficit cognitivo, o que limitava a compreensão das informações fornecidas nas consultas e sobre o uso dos medicamentos. Percebe-se que as dificuldades relacionadas às informações sobre medicamentos compreendem a dificuldade em ler a prescrição médica e as informações na embalagem do medicamento, o que demanda uso de novos métodos para facilitar o entendimento dos pacientes sobre sua farmacoterapia, principalmente no que concerne a tomada de medicamentos. ${ }^{7}$ Foram utilizados diversos recursos como materiais com figuras de sol, lua e alimentos, números, cores e caixas de diferentes formas para auxiliar na compreensão das informações sobre o uso de medicamentos.

Apesar da incipiente literatura cientifica que avalia o uso de pictogramas na prática clínica e a associação da compreensão e aceitabilidade do seu uso entre pessoas com nível inadequado de letramento funcional em saúde, um estudo sugeriu que a utilização de pictogramas pode auxiliar o entendimento adequado das orientações farmacológicas neste grupo de indivíduos, podendo beneficiar principalmente os grupos de menor escolaridade e idosos. ${ }^{18}$

Outro obstáculo para a adesão farmacoterapêutica foi 0 alto custo dos medicamentos em uso, que dificultava o acesso a esses medicamentos. É fundamental assegurar continuidade e garantia do acesso gratuito a medicamentos, uma vez que o custo dificulta e restringe a aquisição por parte da população, refletindo sobre o comportamento do usuário em relação à sua terapia medicamentosa. ${ }^{19}$

O uso de plantas medicinais também surgiu como fator de dificuldade a adesão farmacoterapêutica, uma vez que muitos usuários utilizavam em substituição ao tratamento medicamentoso. A prática do cuidado através de plantas medicinais geralmente se baseia na indicação popular e no conhecimento empírico que muita das vezes são repassados de família a família. Reconhecer o uso destes recursos terapêuticos pelos pacientes é relevante para assegurar que a experiencia de uso dos medicamentos seja a mais efetiva e segura possível pois, a utilização de algumas espécies pode significar riscos potenciais de interações medicamentosas, interferindo no 
metabolismo e na ação de fármacos, resultando em ineficácia terapêutica e risco de toxicidade. ${ }^{20}$

Foram identificados alguns problemas relacionados a farmacoterapia, como omissão e adição de doses pelo usuário, automedicação, armazenamento incorreto de medicamentos e condição clínica sem tratamento. Cenários como este denotam a importância do acompanhamento farmacoterapêutico para a melhoria da farmacoterapia, se constituindo como uma ferramenta fundamental para a redução de erros de medicação e promoção da segurança dos pacientes ${ }^{21}$. Foram fornecidas informações sobre a condição patológica do usuário e seus medicamentos, explicando a sua forma de ação no organismo, os benefícios e possíveis reações na sua utilização além da importância de não abandonar o tratamento proposto pela equipe da unidade.

\section{Considerações finais}

A experiência com o grupo de idosos em polifarmácia evidenciou aspectos relacionados à ocorrência de polifarmácia em idosos e a sua adesão à terapêutica proposta.

Os idosos no geral apresentaram dificuldade de compreensão das orientações para seguimento do projeto terapêutico, bem como falhas no manejo de sua condição de saúde, adesão medicamentosa e déficit de autocuidado, tais como a administração de medicamentos e seu armazenamento. Tais fatores estão relacionados, principalmente, às condições sociais, econômicas, baixa escolaridade e problemas cognitivos.

Ressalte-se que antes da realização dos atendimentos foi necessário o agendamento prévio das visitas domiciliares com os usuários, porém no momento da visita, muitos usuários não foram encontrados em suas residências, postergando o tempo dedicado para essa etapa e com isso a redução do número de participantes do projeto. Um outro ponto a ser analisado foi a dificuldade de acompanhamento da assistente social e da terapeuta ocupacional a todas as visitas devido à baixa carga horária das profissionais na unidade na qual tinham uma alta demanda de atendimentos e reuniões. Com isso, algumas visitas tiveram seu tempo reduzido drasticamente, influenciando nos achados desse projeto.

A inserção do farmacêutico nas atividades clínicas da equipe da unidade foi importante para assegurar a integralidade das ações de saúde, pois a contribuição deste profissional agrega conhecimentos e habilidades importantes para o atendimento das necessidades relacionadas à farmacoterapia dos pacientes. Apesar da contribuição do trabalho farmacêutico descrita neste relato não foi possível abordar os desfechos clínicos resultantes das intervenções farmacêuticas realizadas com os usuários. É relevante conduzir estudos que sejam capazes de demonstrar tais resultados.

Depreende-se, a partir desta experiência descrita, que a prevenção quaternária no idoso em polifarmácia pressupõe a atuação próxima à realidade do sujeito, intervindo em seu contexto social, econômico e cultural, a ampliação da clínica com uso de ferramentas como o apoio matricial, que propicia um aprendizado desmedicalizante, a garantia da continuidade do cuidado com base em evidências científicas, e o acolhimento e vínculo estabelecido com a equipe de referência da área de abrangência.

\section{Referências}

1. Almeida AN, Almeida ORA, Capriata SAR, Cândido SAM, Chaves CJD, Cegati SL. Prevalência e fatores associados à polifarmácia entre os idosos residentes na comunidade. Revista Brasileira de Geriatria e Gerontologia, 20(1):143-153, 2017. Disponível em: https://www.redalyc.org/pdf/4038/4038507070 15.pdf> Acesso em 29 de setembro de 2020.

2. Jacinto LAO. Atividade Física, Depressão e Qualidade de Vida em Pessoas Idosas do Estado do Amazonas [dissertação]. Manaus: Universidade Federal do Amazonas. 2020. Disponível em: https://digituma.uma.pt/bitstream/10400.13/27 87/1/MestradoLuizJacinto.pdf> Acesso em 29 de setembro de 2020.

3. Miranda GMD, Mendes ACG, Silva ALA. O envelhecimento populacional brasileiro: desafios e consequências sociais atuais e futuras. Revista Brasileira de Geriatria e Gerontologia, Rio de Janeiro, 19(3):507-519, Junho, 2016. Disponível em: https://doi.org/10.1590/180998232016019.150140>Acesso em 14 de outubro de 2020.

4. Veras RP, Oliveira M. Envelhecer no Brasil: a construção de um modelo de cuidado. Ciência saúde coletiva, Rio de Janeiro, 23(6) 1929-1936, jun. 2018. Disponível em: http://dx.doi.org/10.1590/1413-

81232018236.04722018> Acesso em 14 de outubro de 2020.

5. IBGE - INSTITUTO BRASILEIRO DE GEOGRAFIA E ESTATÍSTICA. Acesso em 09 de outubro de 2020. Rio de Janeiro. Disponível em: 
https://agenciadenoticias.ibge.gov.br/media/co $\mathrm{m}$ mediaibge/arquivos/0ea24a6209e3a7b9ac95 6 b645dabecf4.xls. Acesso em 14 de outubro de 2020.

6. Malta DC, Bernal RTI, Lima MG, Araújo SSC, Silva, MMA, Freitas MIF. Doenças crônicas não transmissíveis e a utilização de serviços de saúde: análise da Pesquisa Nacional de Saúde no Brasil. Revista de Saúde Pública. 2017;51(Supl). Disponível em: https://doi.org/10.1590/s15188787.2017051000090> Acesso em 20 de setembro de 2020.

7. Martins NFF, Abreu DPG, Silva BT, Semedo DSRC, Pelzer MT, Lenczak FS. Letramento funcional em saúde e adesão à medicação em idosos: revisão integrativa. Revista Brasileira de Enfermagem [Internet]. 2017;70(4):868-74. Disponível em: https://www.scielo.br/scielo.php?pid=S0034-

71672017000400868\&script=sci arttext\&tlng=pt $>$ Acesso em 29 de setembro de 2020. DOI: http://dx.doi.org/10.1590/0034-7167-2016-0625

8. Sousa, WEA. Funcionalidade Familiar De Idosos Com Diabetes Mellitus [dissertação]. São Luis (MA): Universidade Federal do Maranhão; 2017. Disponível em> https://monografias.ufma.br/ispui/bitstream/12 3456789/2029/1/WalanaSousa2017.pdf> Acesso em 29 de setembro de 2020.

9. Pereira KG, Peres MA, lop $D$, Boing $A C$, Aziz, M, D’orsi, E. Polifarmácia em idosos: um estudo de base populacional. Revista Brasileira de Epidemiologia, São Paulo, 20(2): 335-344, junho $2017 . \quad$ Disponível em: http://dx.doi.org/10.1590/1980-

5497201700020013> Acesso em: 14 de outubro de 2020 .

10. Araújo, NC. Estudo De Coorte Sobre O Uso De Medicamentos Potencialmente Inapropriados Em Idosos. Goiânia, 2020. Disponível em: https://repositorio.bc.ufg.br/tede/bitstream/ted e/10733/3/Disserta\%c3\%a7\%c3\%a3o\%20-

\%20Natacha\%20Christina\%20de\%20Ara\%c3\%bai o\%20-\%202020.pdf> Acesso em 29 de setembro de 2020 .

11. Sales AS, Sales MGS, Casotti CA. Perfil farmacoterapêutico e fatores associados à polifarmácia entre idosos de Aiquara, Bahia, em 2014. Epidemiologia e Serviços de Saúde, 26(sn);121-132, 2017. Disponível em: http://dx.doi.org/10.5123/s1679-

49742017000100013> Acesso em 16 de março de 2021.

12. Tesser,CD. Por que é importante a prevenção quaternária na prevenção? Rev. Saúde Pública. v. 51, n..116, p. 1-9, 2017.Disponível em: http://www.rsp.fsp.usp.br/artigo/por-que-eimportante-a-prevencao-quaternaria-naprevencao/> Acesso em 16 de março de 2021. 13. Secoli, SR. Polifarmácia: interações e reações adversas no uso de medicamentos por idosos. Revista Brasileira de Enfermagem. [online]. 2010, 63(1):136-140. Disponível em: https://doi.org/10.1590/S0034-

71672010000100023> Acesso em 01 de outubro de 2020.

14. Melo DO, Castro LLC. A contribuição do farmacêutico para a promoção do acesso e uso racional de medicamentos essenciais no SUS. Ciênc. saúde coletiva, Rio de Janeiro , v. 22, n. 1, p. 235-244, Jan. 2017. Disponível em: http://www.scielo.br/scielo.php?script=sci artte xt\&pid=S141381232017000100235\&Ing=en\&nrm =iso Acesso em 16 de Março de 2021.

15. Cecilio LCO, Reis AAC. Apontamentos sobre os desafios (ainda) atuais da atenção básica à saúde. Caderno de Saúde Pública. 2018; 34(8):e00056917. DOI: 10.1590/0102$311 \times 00056917>$ Acesso em 10 de outubro de 2020.

16. Daltro MR, Faria, AA. Relato de experiência: Uma narrativa científica na pósmodernidade. Estudos e Pesquisas em Psicologia, Rio de Janeiro, v.19, n 1, p.223-237, 2019. Disponível em: < https://www.epublicacoes.uerj.br/index.php/revispsi/article/vie w/43015/29664> Acesso em 03 de dezembro de 2019.

17. Ramos LR, Tavares NUL, Bertoldi AD, Farias MR, Oliveira MA, Luiza VL, et al. Polifarmácia e polimorbidade em idosos no Brasil: um desafio em saúde pública. Rev Saude Publica. 2016;50(supl 2):9s.

18. Neto JAC, Costa LA, Estevanini GM, Alves $\mathrm{GL}$, Ferreira $\mathrm{R}$ E. Uso de pictogramas na prescrição médica e letramento funcional em saúde. Brazilian Journal of Surgery and Clinical. 23(2)51-57.

19. Fritzen JS, Motter FR, Paniz VMV. Acesso regular e adesão a medicamentos do componente especializado assistência farmacêutica. Rev Saude Publica. 2017;51:109. Disponível em: http://www.rsp.fsp.usp.br/wpcontent/uploads/articles xml/0034-8910-rspS1518-87872017051006932/0034-8910-rspS1518-87872017051006932-pt.x83745.pdf. Acesso em 19 de Março de 2021.

20. Scheid T, Fajardo AP. Uso de plantas medicinais por idosos adscritos à atenção primária em Porto Alegre/RS e potenciais interações planta-medicamento. Rev Fitos. Rio de Janeiro. 2020; 14(1): 103-117. e-ISSN 
2446.4775. Disponível em:

http://revistafitos.far.fiocruz.br/index.php/revist a-fitos/article/view/801 Acesso em 19 de Março de 2021.

21. Silva L. Elaboração de método de acompanhamento farmacoterapêutico em uma unidade de referência em doenças infecciosas: contribuição para a segurança do paciente. Rio de Janeiro, 2017. 76f. Dissertação [Mestrado Profissional em Pesquisa Clínica] -Instituto Nacional de Infectologia Evandro Chagas. Disponível em> https://www.arca.fiocruz.br/bitstream/icict/3342 5/2/lion silva ini mest \%202017.pdf Acesso em 19 de Março de 2021

\section{Endereço para Correspondência}

Amanda Maria Villas Bôas Ribeiro

Rua Basílio da Gama, s/n - Canela,

Salvador - BA, Brasil

CEP: $40110-040$

E-mail: amanda marias@yahoo.com.br

Recebido em 16/03/2021

Aprovado em 22/05/2021

Publicado em 27/09/2021 